Revue des patrimoines

$43 \mid 2021$

Des écoles d'art académiques aux écoles d'art : des collections et des lieux, un patrimoine à valoriser

\title{
Exposer le patrimoine des écoles d'art en Europe : un intérêt résolument contemporain
}

Exhibiting the patrimony of art schools in Europe: a typical European topic

\section{Morwena Joly-Parvex}

\section{(2) OpenEdition}

\section{Journals}

Édition électronique

URL : http://journals.openedition.org/insitu/29326

DOI : $10.4000 /$ insitu.29326

ISSN : $1630-7305$

Éditeur

Ministère de la Culture

Référence électronique

Morwena Joly-Parvex, « Exposer le patrimoine des écoles d'art en Europe : un intérêt résolument contemporain », In Situ [En ligne], 43 | 2021, document 14, mis en ligne le 12 janvier 2021, consulté le 14 janvier 2021. URL : http://journals.openedition.org/insitu/29326 ; DOI : https://doi.org/10.4000/ insitu. 29326

Ce document a été généré automatiquement le 14 janvier 2021.

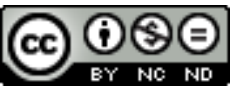

In Situ Revues des patrimoines est mis à disposition selon les termes de la licence Creative Commons Attribution - Pas d'Utilisation Commerciale - Pas de Modification 4.0 International. 


\title{
Exposer le patrimoine des écoles d'art en Europe : un intérêt résolument contemporain
}

\author{
Exhibiting the patrimony of art schools in Europe: a typical European topic
}

\author{
Morwena Joly-Parvex
}

Les écoles d'art qui ont été fondées dans toute l'Europe, du XVII ${ }^{\mathrm{e}}$ au $\mathrm{xVIII}^{\mathrm{e}}$ siècle, formulent aujourd'hui les mêmes questions: comment mieux connaître et mieux valoriser leur patrimoine et à quelles fins ? De l'ouverture d'un nouvel espace muséal à la Royal Academy de Londres en 2016 à celle de la collection anatomique de Dresde en 2018, de colloques en projets de recherche autour des nombreuses gypsothèques allemandes, force est de constater que partout en Europe, l'intérêt se porte sur ces collections atypiques dans l'univers muséal, où les chefs-d'œuvre se mêlent à quantité de dessins et de plâtres souvent mal connus ou à des collections redondantes. Exposer ces collections, c'est donner à voir l'histoire de la formation des artistes, la manière dont l'art s'élabore, parfois avec des injonctions contradictoires ou des approches variées. La difficulté tient bien souvent à ce que les collections pédagogiques ont été conservées de manière plus ou moins aléatoire, et que les divers legs, venus enrichir certains fonds d'académies, viennent troubler la cohérence initiale, sans compter l'importance prise par les plâtres gréco-romains principalement, qui peinent à s'harmoniser avec notre vision contemporaine de la création.

$2 \mathrm{Si}$, encore aujourd'hui, les artistes s'entraînent à copier dans les musées, le principe d'une formation par l'exemple et d'un apprentissage fondé sur l'observation des modèles anciens semble de plus en éloignée de l'image de l'artiste absolument singulier et original qui s'est imposée aujourd'hui. Si le musée s'est fait école de fait, l'école doitelle se faire pour autant musée ? La nature des expositions permanentes ne peut y être exactement identique à celle du musée et cette difficulté muséographique est aujourd'hui de nouveau questionnée, d'autant qu'elle doit aussi tenir compte des liens entre patrimoine et création contemporaine, cette dernière étant au cœur de l'activité des écoles d'art. 
3 Les réponses apportées dans les grandes académies européennes diffèrent en raison de leurs histoires, confondues puis séparées de l'institution muséale, et parfois de la section archéologique de l'université. En parcourant l'Europe, de Londres aux grandes villes allemandes, de Madrid à Vienne et jusqu'à Saint-Pétersbourg, une typologie s'esquisse assez naturellement. Il y a tout d'abord le cas des écoles d'art nationales qui se sont dotées d'un département «musée» comme à Madrid, Vienne ou SaintPétersbourg, à la faveur de circonstances historiques singulières. À l'opposé, les écoles d'art italiennes et allemandes, qui se sont concentrées aujourd'hui sur leur vocation pédagogique, n'ont dans leur majorité pas conservé leurs collections patrimoniales en leur sein. Les galeries des académies sont devenues des institutions autonomes à partir du milieu du XIX ${ }^{e}$ siècle dans l'Italie unifiée, tandis qu'en Allemagne, les écoles d'art ont massivement opté pour une claire distinction entre activité pédagogique et muséale, sans doute en raison de la naissance précoce de musées sur le territoire. Enfin, le cas de la Royal Academy de Londres est particulièrement intéressant: une partie de sa collection est désormais exposée de manière permanente et cohabite avec une politique d'expositions temporaires sur l'art contemporain ou l'art ancien. Trois manières fort différentes d'aborder le patrimoine des écoles d'art, en fonction du prestige des legs historiques mais aussi des possibilités offertes par le bâtiment ou encore de l'environnement muséal proche. Ce panorama montre la diversité des options scientifiques et muséographiques prises et permet d'esquisser des pistes de réflexion pour la mise en valeur du patrimoine des écoles d'art: l'intérêt de l'amateur pour la formation artistique est aussi celui d'un public plus large, pour qui le « devenir artiste » demeure une question fascinante, au point de devenir régulièrement un sujet d'exposition en soi. Le patrimoine des écoles d'art peut-il participer à éclairer ce mystère de l'apprentissage de la création?

\section{Madrid, Vienne, Saint-Pétersbourg : un patrimoine, un musée}

4 Les écoles académiques de Madrid, Vienne et Saint-Pétersbourg constituent un groupe bien distinct en raison de la richesse historique de leurs collections. Ces institutions ont ainsi pu se doter d'un musée propre dans la mesure où elles conservent des collections de peinture et de sculpture suffisamment prestigieuses pour être présentées de manière permanente au public. Ces musées sont appréciés des visiteurs en raison de leur taille humaine et d'une qualité de visite qui n'existe que rarement dans les grands musées nationaux. Néanmoins, ces musées peinent à obtenir une visibilité à côté de grands musées comme le Prado, le Kunsthistorisches Museum ou l'Ermitage. Une " œuvre phare" offre en général le motif de la visite, que ce soit Le Printemps de Giuseppe Arcimboldo ou la collection de peintures de Francisco de Goya à l'Académie de Madrid ou encore Le Jugement dernier de Jérôme Bosch à celle de Vienne. Seul le musée de Saint-Pétersbourg ne bénéficie d'aucune œuvre particulièrement remarquable susceptible d'attirer l'attention des visiteurs, une grande partie de sa prestigieuse collection ayant été transférée au musée de l'Ermitage après 1917, et peine à s'imposer au sein de l'offre culturelle foisonnante de la ville.

5 Ces importantes académies fondées dans les capitales européennes au XVIII siècle ont en commun d'être des créations royales ou princières destinées à diffuser la représentation de la gloire du souverain, que l'idée de la fondation émane entièrement 
des cercles politiques comme en Russie avec la création de l'Académie impériale des beaux-arts de Saint-Pétersbourg en 1757 ou que l'initiative en revienne à des artistes comme à Madrid avec l'Académie de Saint-Ferdinand en 1752, ou avec l'Académie des beaux-arts de Vienne en 1692. Ces créations académiques se sont ainsi accompagnées de dons prestigieux, qui forment une véritable collection muséale, mais dont le lien avec le sujet même de la formation de l'artiste est quelque peu perdu au profit d'une mise en valeur strictement muséale des collections.

6 Le musée qui fonctionne le plus indépendamment de l'activité pédagogique est celui de l'Académie des beaux-arts de Vienne (Akademie der bildenden Künste Wien) [fig. 1]. Il s'agit d'une galerie de peinture de niveau international qui n'a plus guère de lien avec la formation des artistes, si ce n'est qu'elle offre un cadre muséal à sa riche collection. La galerie d'art se déploie au deuxième étage - le bâtiment abritant d'autres services de l'école - et conserve 1500 tableaux, dont seule une petite partie peut être accrochée.

Figure 1

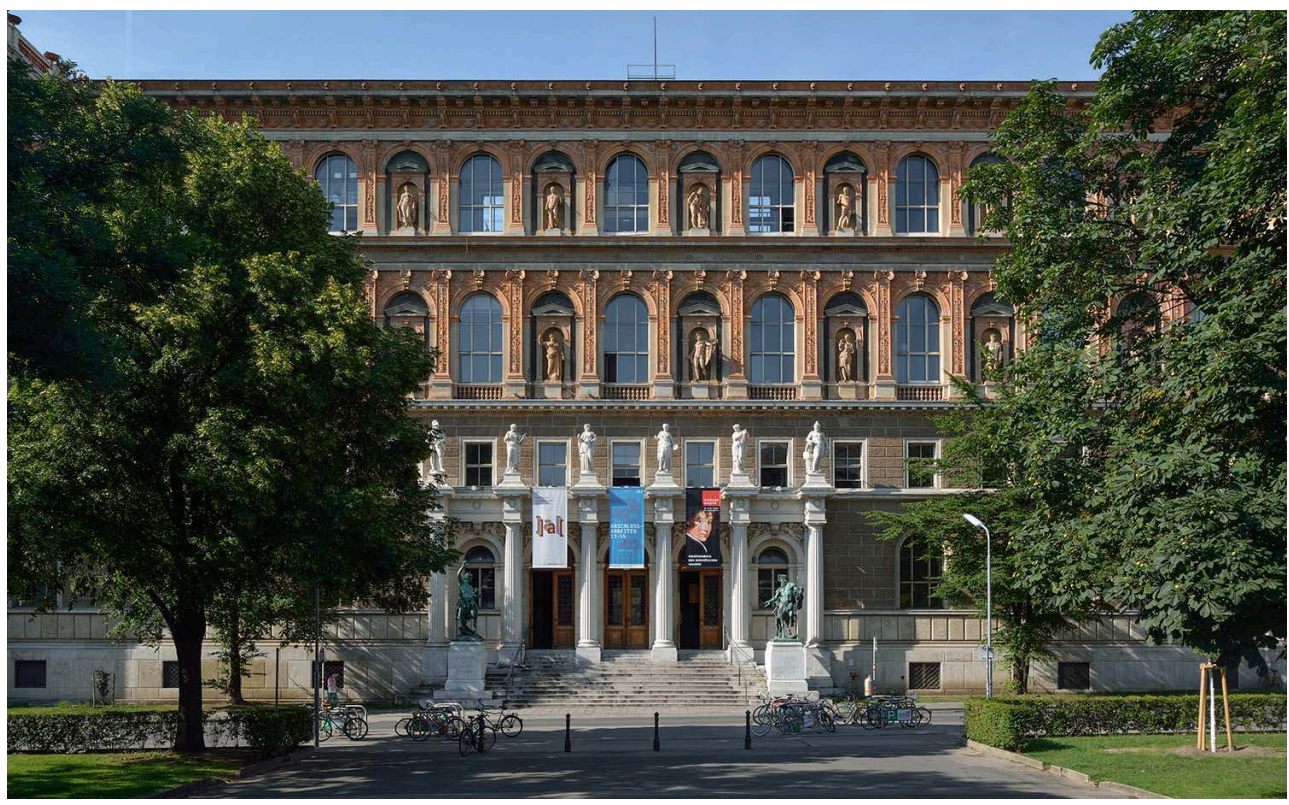

Académie des beaux-arts de Vienne.

(c) Peter Hass / reproduction Wikipédia CC BY-SA 3.0.

Fondée en 1692 à l'initiative du sculpteur Peter Strudel (1660-1714), l'Académie ne possède initialement aucune collection en dehors des œuvres de ses professeurs et de celles primées des élèves, avant que le comte Anton Franz von Lamberg-Sprinzenstein, diplomate des Habsbourg à Turin et à Naples, ne lègue en 1822 sa fameuse collection de peintures européennes. Une galerie est alors créée, en conformité avec les termes du legs, qui stipulent que la collection doit être ouverte au public - devenant ainsi le premier musée d'art en Autriche - et conservée à des fins pédagogiques. L'activité muséale a ainsi été intégrée aux statuts de l'Académie. Ce don de 800 peintures, qui comprend le triptyque du Jugement dernier de Jérôme Bosch mais aussi des tableaux et esquisses peintes de Pierre-Paul Rubens ainsi qu'une riche collection de tableaux hollandais du XVII siècle, hisse d'emblée la galerie au niveau international' ${ }^{1}$. L'Académie conserve également le célèbre autoportrait d'Anton Van Dyck, un ensemble de peintures allemandes - et pas moins de six tableaux de Lucas Cranach l'Ancien- 
italiennes, flamandes et anglaises, et autrichiennes. Les écoles de peinture italiennes sont représentées par Sandro Botticelli, Titien, Giambattista Tiepolo et Francesco Guardi, pour n'en citer que les plus connus. Aujourd'hui, l'œuvre de Jérôme Bosch est régulièrement mise en avant dans la promotion des collections à destination du grand public en raison de la rareté de ses œuvres.

8 La collection de l'Académie s'est par la suite encore enrichie, notamment avec la donation, par le prince Jean II de Liechtenstein (1840-1929) d'une riche collection de peintures italiennes. L'ensemble s'expose aujourd'hui dans une Gemäldegalerie, un bâtiment spécialement ouvert à cette fin en 1877 , sur la Schillerplatz. C'est à l'époque du transfert de la collection dans ce nouvel édifice qu'une sélection drastique des œuvres exposées a été réalisée, reléguant au second plan les œuvres moins prestigieuses offertes par les académiciens et ordonnant l'accrochage des collections par écoles ${ }^{2}$. Le dernier don majeur fait par un mécène date de 1957, enrichissant de nouveau le musée d'une collection de peintures, principalement de l'école néerlandaise.

Ces circonstances historiques ont contribué à mettre au second plan les collections plus strictement pédagogiques. Certes, la salle anatomique de l'école est demeurée intacte et préservée depuis sa création et la collection historique de plâtres, qui compte environ 450 pièces, est exposée sous forme de dépôts dans l'atelier de l'Académie des beauxarts. Mais seul L'Écorché « au bras levé »w de Jean-Antoine Houdon voisine avec la prestigieuse collection de peinture. À l'ouverture du nouveau musée en 1877, la gypsothèque est exposée au centre du bâtiment, de manière chronologique car au-delà du fonds gréco-romain, toutes les périodes ont été couvertes depuis la création de l'institution. Cette collection a été peu à peu écartée du circuit de visite traditionnel [fig. 2], au profit de la collection de peinture. On voit clairement évoluer les motifs de visite en lisant les guides de voyage du début du XIX ${ }^{e}$ siècle à nos jours : si, en 1808, on vient voir les collections de l'Académie avant le legs Lamberg, c'est pour y admirer les plâtres d'antiques ${ }^{3}$. Après la donation, les guides recommandent la visite de la galerie pour y contempler les chefs-d'œuvre de l'école allemande et flamande ${ }^{4}$ car ce sont eux qui conféraient à la collection Lamberg une renommée européenne du vivant même du comte. 
Figure 2

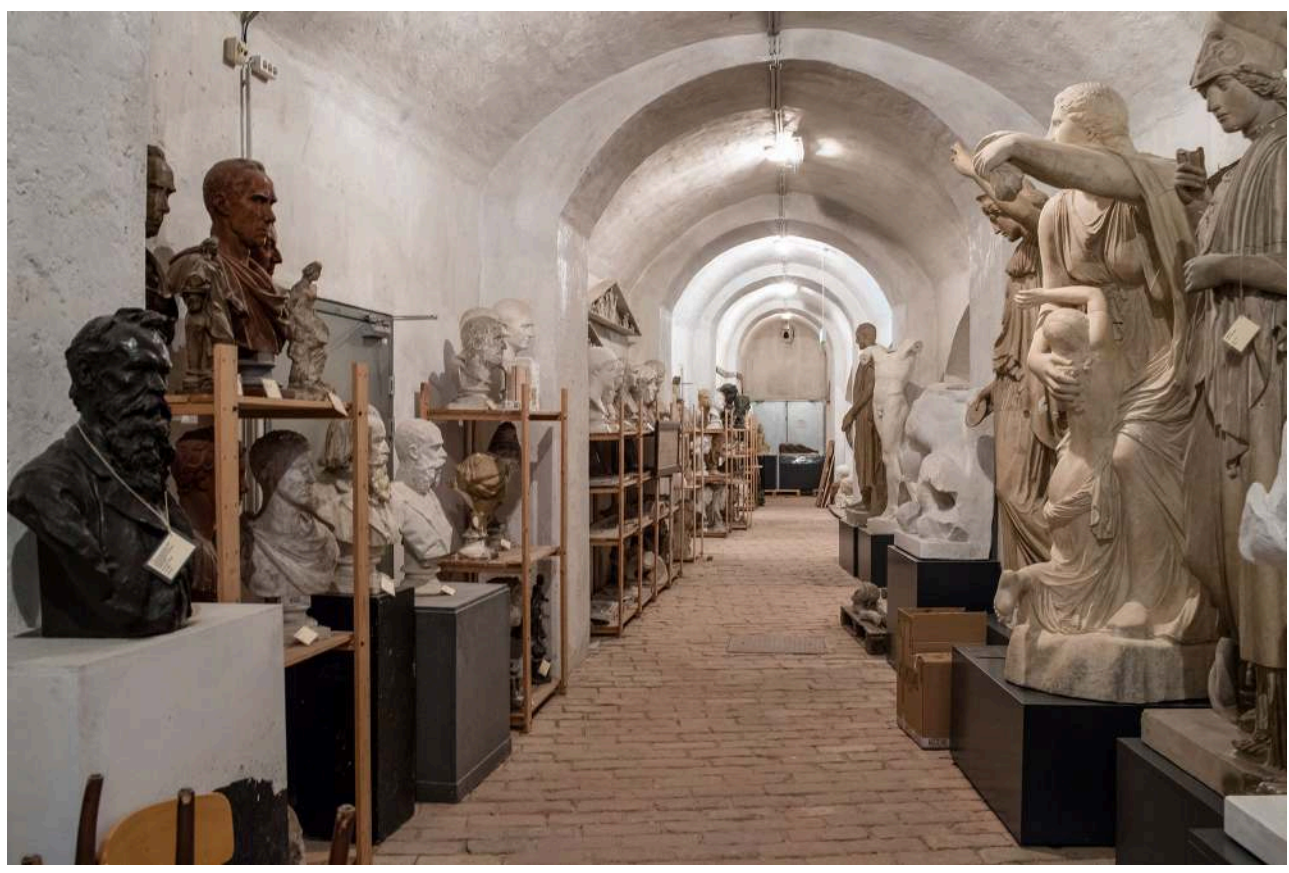

Vue de la galerie des plâtres de l'Académie de Vienne.

(C) Photographe inconnu (Akademie der Bildenden Künst).

10 Si la logique muséale domine d'emblée en raison des intentions de son donateur, le discours sur l'histoire de la pédagogie artistique est parfois mis en valeur, car l'Académie de Vienne a joui d'une grande notoriété en Europe. La richesse de la donation permet de mettre en avant le processus de la création artistique dans le cadre d'expositions temporaires. Les nombreuses esquisses peintes de Rubens sont intégrées dans un discours global sur la manière dont le peintre flamand s'inspire des anciens modèles car l'Académie possède quatre esquisses exécutées au cours de ses années de formation en Italie au début du XVII ${ }^{e}$ siècle, influencées par la " grande maniera » de l'art baroque italien. L'autoportrait de Van Dyck [fig. 3] tisse également un lien fort avec l'activité de l'Académie, parce qu'il s'agit du portrait d'un jeune artiste, au regard déterminé, mais aussi interrogatif sur sa postérité. Mais dans l'ensemble, le musée peine à établir des liens historiques avec la formation des artistes dans la mesure où une sélection drastique des œuvres d'élèves et de professeurs a été opérée au moment du transfert de la galerie dans son nouveau bâtiment, en 1877. Aujourd'hui, les expositions d'art ancien et d'art contemporain se mêlent, et la figure de Jérôme Bosch est fréquemment convoquée à des fins de confrontation avec la création moderne et contemporaine. 
Figure 3

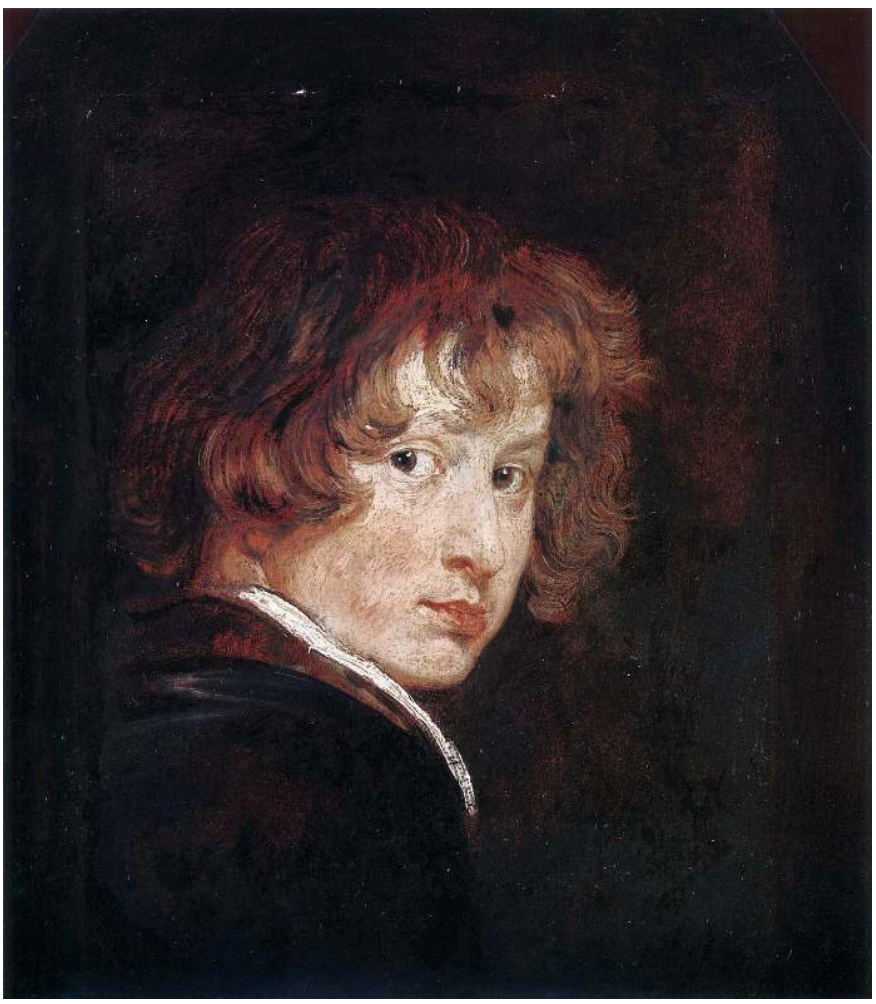

Autoportrait, Anthonis van Dyck (1599-1641), vers 1615, huile sur bois $(26 \times 20 \mathrm{~cm})$ conservée à I'Akademie der Bildenden Künst, Vienne.

Reproduction Akademie der Bildenden Künst. 
Figure 4

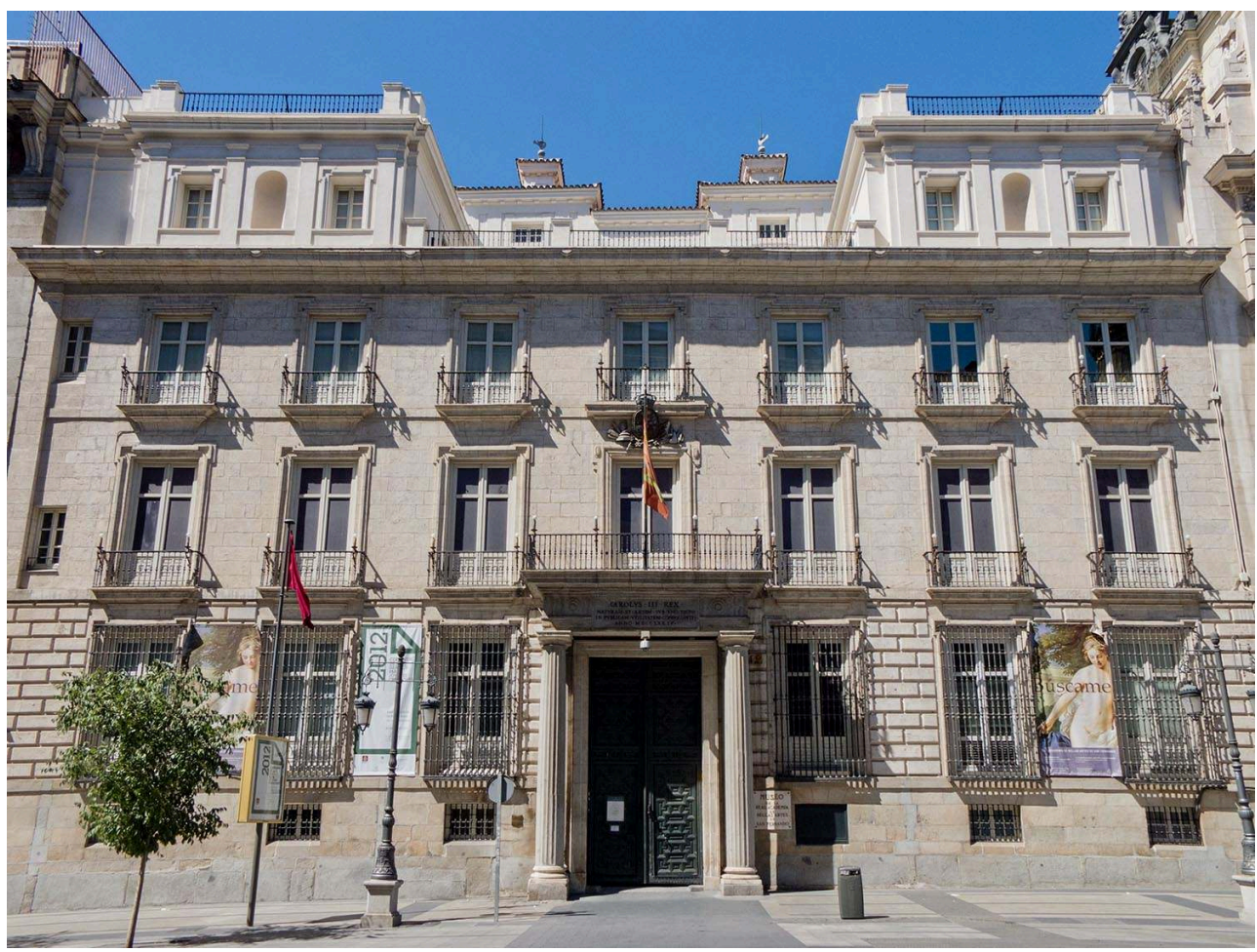

Académie des Beaux-Arts de San Fernando de Madrid.

(c) Carlos Delgado / reproduction Wikipédia CC BY-SA 3.0.

11 Le musée de l'Académie des beaux-arts de Madrid [fig. 4] a été quant à lui créé à la faveur du succès des expositions de ses étudiants à la fin du XVIII ${ }^{e}$ siècle et en raison de son exceptionnelle collection, rassemblant des dons de la famille royale et des œuvres saisies / collectées dans les monastères espagnols lors de la suppression des ordres religieux. Définie par le roi Philippe V, petit-fils de Louis XIV, l'Académie des beauxarts de Madrid est fondée en $1752^{5}$ sous l'égide de son fils le roi Ferdinand VI, dont elle porte le nom (Academia de San Fernando). L'institution triple le nombre de ses élèves de 1758 à 1800, pour s'établir à un millier, et finance des séjours à l'étranger depuis 1758, notamment à Paris et à Rome. L'histoire des collections de l'Académie a été marquée par deux personnalités artistiques majeures: les peintres Anton Raphaël Mengs et Francisco de Goya. Ce dernier, déjà académicien depuis 1780, devient le directeur adjoint de l'Académie de 1785 à 1792 et demeure l'artiste le plus présent avec treize tableaux, dont son célèbre autoportrait au chevalet [fig. 5]. Mengs a, en ce qui le concerne, laissé son empreinte dans les collections en léguant une partie de ses plâtres d'antiques, collection admirée et connue de l'Europe entière à la fin du xvIII ${ }^{e}$ siècle [fig. 6]. 
Figure 5

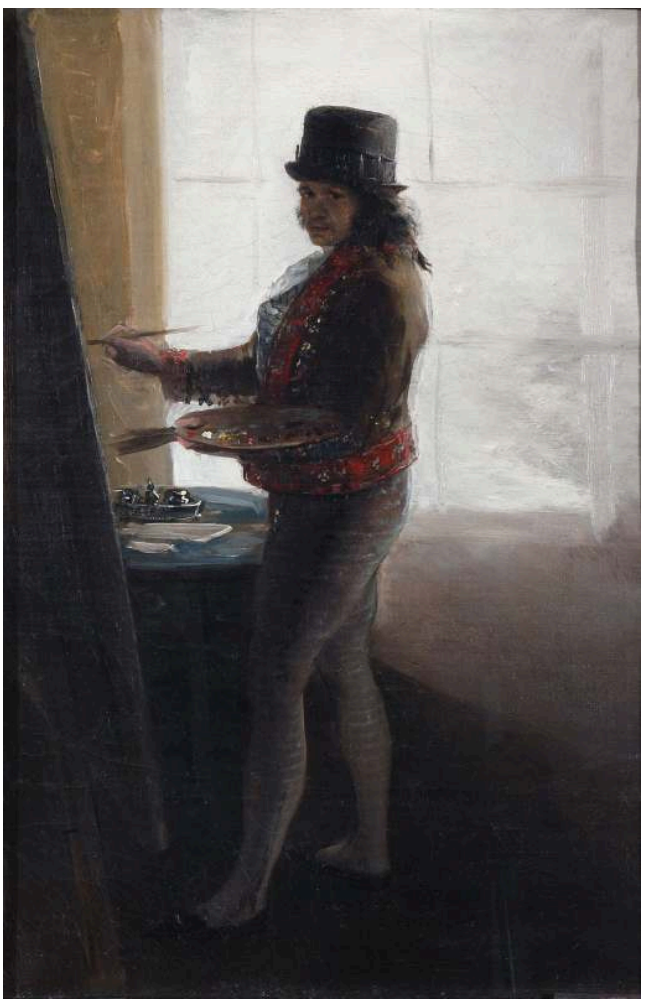

Autoportrait au chevalet, Francisco de Goya (1746-1828), vers 1785, huile sur toile (40 x $27 \mathrm{~cm}$ ) conservée à la Real Academia de Bellas Artes de San Fernando (inv. 1166), Madrid.

Reproduction Real Academia de Bellas Artes de San Fernando. 
Figure 6

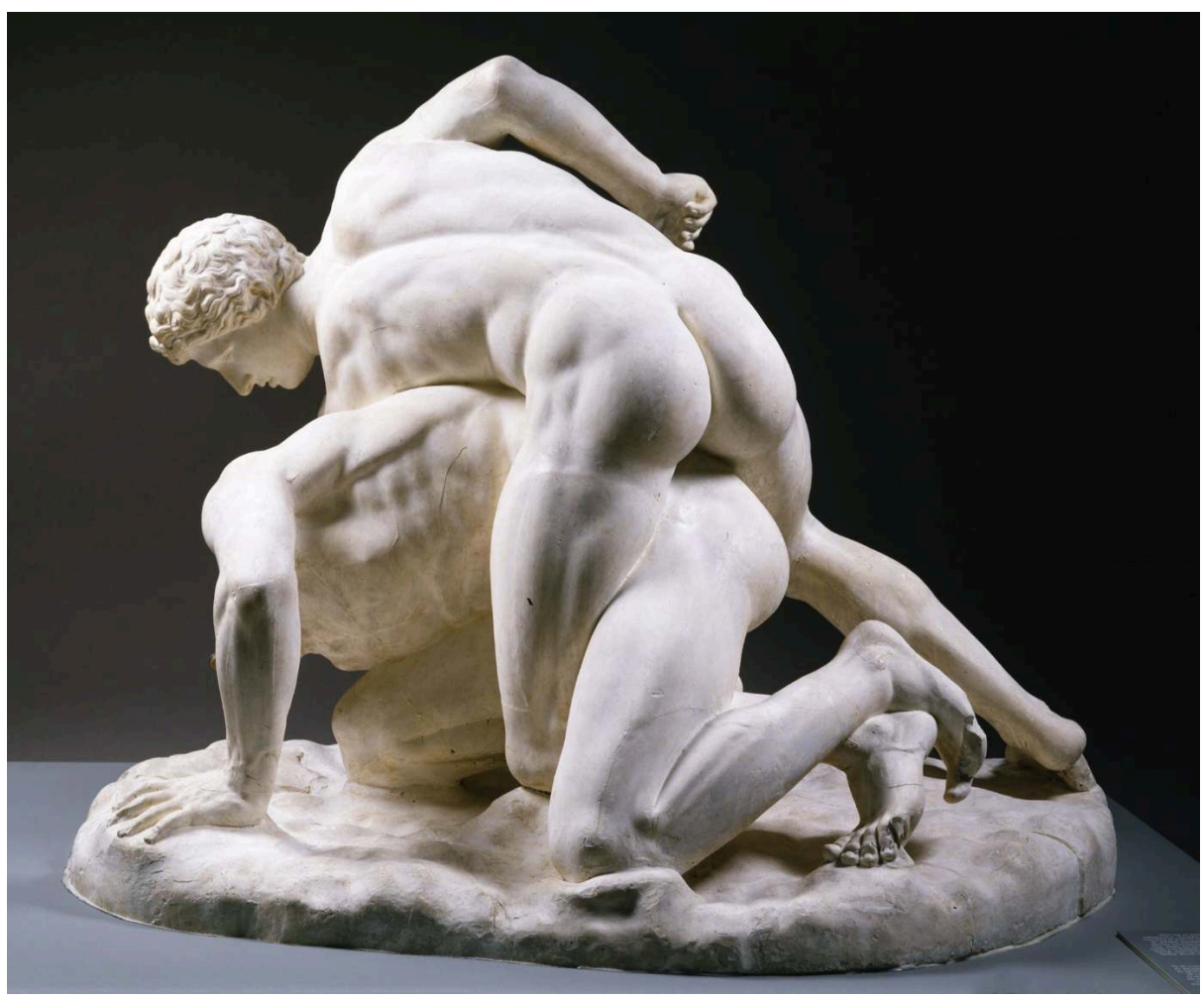

Moulage en plâtre des Lutteurs de Florence, 1770, issu de la collection d'Anton Raphael Mengs (1728-1779). Groupe original découvert en 1583 à Rome (conservé aujourd'hui au musée des Offices de Florence), dont le moulage est donné par Anton Rafael Mengs à Carlos III pour son Académie. Plâtre (h. 100 xI. 117 x p. 80 cm) conservé à la Real Academia de Bellas Artes de San Fernando (inv. V-022), Madrid.

(C) Photographe inconnu (Real Academia de Bellas Artes de San Fernando).

D'abord conçue pour être exclusivement à l'usage des artistes, l'Académie décide d'offrir au public la possibilité de visiter les collections de l'école ${ }^{6}$ en 1793 , décision qui s'impose face à la demande car la capitale espagnole ne possède pas encore de musée. Devant ce succès et la volonté d'affirmer l'existence d'une école nationale de peinture après l'invasion française, Ferdinand VII a même voulu doter l'Académie d'un nouveau bâtiment conçu pour être un musée de peinture, sculpture et gravure en donnant un nouveau palais au corps académique en $1814^{7}$. En définitive, la volonté politique s'incarne avec l'ouverture du musée du Prado en $1819^{8}$.

13 Outre les œuvres que chaque académicien doit donner à son entrée, les collections ont été enrichies au fil des siècles par d'importants legs, couvrant les arts $d u x^{e}$ au $\mathrm{XX}^{\mathrm{e}}$ siècle. Près de 1400 peintures, 15000 dessins et estampes, mais aussi 570 sculptures forment aujourd'hui la collection du musée.

14 La qualité et le nombre d'œuvres de l'école espagnole fonde la cohérence du fonds : aux tableaux de Francisco de Goya s'ajoutent des tableaux de Velázquez, de José de Ribera, de Francisco de Zurbarán, du Greco, de Bartolomé Estéban Murillo ou de Joaquín Sorolla. La collection de sculptures permet aussi de présenter l'évolution de la sculpture espagnole du xvII ${ }^{\mathrm{e}}$ siècle à nos jours, avec des œuvres de Manuel Francisco Álvarez de la Peña (1727-1797), Mariano Benlliure (1862-1947), Pablo Gargallo (1881-1934) et Pablo Picasso. La collection exposée permet de mettre en relief le passage du rococo, cultivé par les artistes italiens installés en Espagne, au 
néoclassicisme sous l'influence du sculpteur Felipe de Castro (1711-1775) et du peintre Anton Raphaël Mengs. Grâce à une politique d'acquisition cohérente et suivie, à travers les dons et legs faits à l'Académie au cours des siècles, le musée se consacre surtout à l'évocation de l'art espagnol, et expose, sans en expliquer la cohérence initiale au sein de l'Académie, un très beau fonds de peintures italiennes et flamandes des $\mathrm{XVI}^{\mathrm{e}}$ et $\mathrm{XVII}^{\mathrm{e}}$ siècles avec les œuvres de Pierre-Paul Rubens, de Domenico Zampieri dit Le Dominiquin, de Giovanni Bellini, Leandro Bassano, Luca Giordano et le seul tableau de Giuseppe Arcimboldo conservé en Espagne [fig. 7].

Figure 7

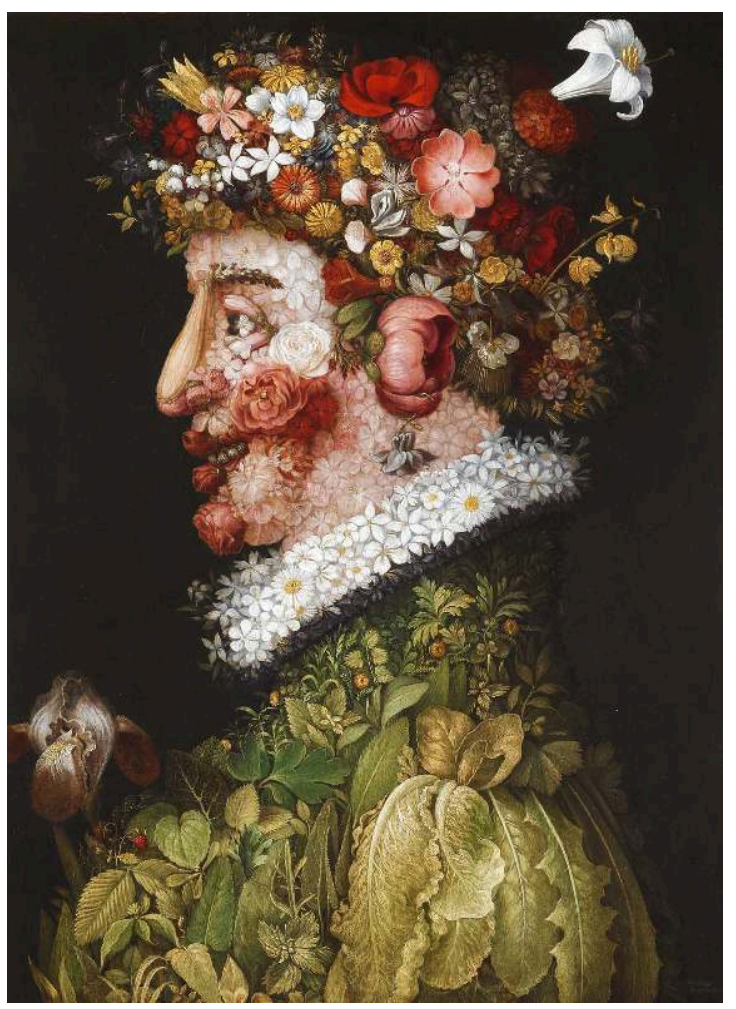

La Primavera, Giuseppe Arcimboldo (1527-1593), 1563, huile sur panneau de bois (66 × $50 \mathrm{~cm}$ ) conservée à la Real Academia de Bellas Artes de San Fernando (inv. 0606).

Reproduction Real Academia de Bellas Artes de San Fernando.

15 Si le palais Goyeneche, où se déploie le musée, offre un panorama relativement complet de l'art espagnol, c'est au détriment de la vocation pédagogique initiale du fonds. Le dessin d'après l'antique est bien évoqué avec la présentation de la collection de plâtres d'antiques qui appartenait à Anton Raphaël Mengs - que l'Académie reçoit en legs en 1778 - mais sans que celle-ci soit confrontée à la pédagogie de Francisco de Goya, dont la méthode d'enseignement mettait en avant l'étude d'après le modèle vivant. De même, la riche collection de dessins, où dominent les artistes italiens et espagnols ${ }^{9}$ n'est pas réellement valorisée. La logique muséale a ainsi conduit le discours de médiation à occulter la spécificité de la collection académique.

Les expositions alternent art ancien et création contemporaine, avec des incursions dans le domaine de la mode, et le discours historique liant le musée et l'école d'art est assez discret. L'activité muséale fonctionne de manière autonome, appuyée par un 
atelier de moulage et de chalcographie à vocation commerciale - entre antique et œuvre gravé de Goya.

À Saint-Pétersbourg, un musée est constitué de fait en même temps que l'Académie impériale des beaux-arts (1757). L'Académie possède en effet dès ses débuts sa propre collection d'œuvres d'art, dont une exceptionnelle collection de plâtres d'antiques envoyés de Rome en $1758^{10}$, complétant la collection des morceaux de réception, les dons de professeurs ou les grands prix d'étudiants, ainsi que des dons de mécènes appartenant à la famille impériale. Les œuvres les plus précieuses ont été offertes par le premier conservateur de l'Académie, le comte Andreï Petrovitch Shuvalov, élu membre honoraire de l'Académie en 1758, et dont le palais accueille l'institution nouvellement créée. Les inventaires mentionnent dans sa donation, aujourd'hui dispersée, les noms prestigieux de Raphaël, Véronèse, Rubens. La bibliothèque de l'Académie n'est pas en reste, offrant dès la fin du XVIII ${ }^{e}$ siècle la consultation de plus de 1500 volumes in-folio, 4500 estampes et 7000 dessins. Après l'accession au trône de Catherine II, ce projet éducatif bénéficie d'un second souffle, l'impératrice lui ayant accordé un "privilège " autorisant la construction d'un grand bâtiment de style néoclassique sur l'île Vassilievski [fig. 8, fig. 9].

Figure 8

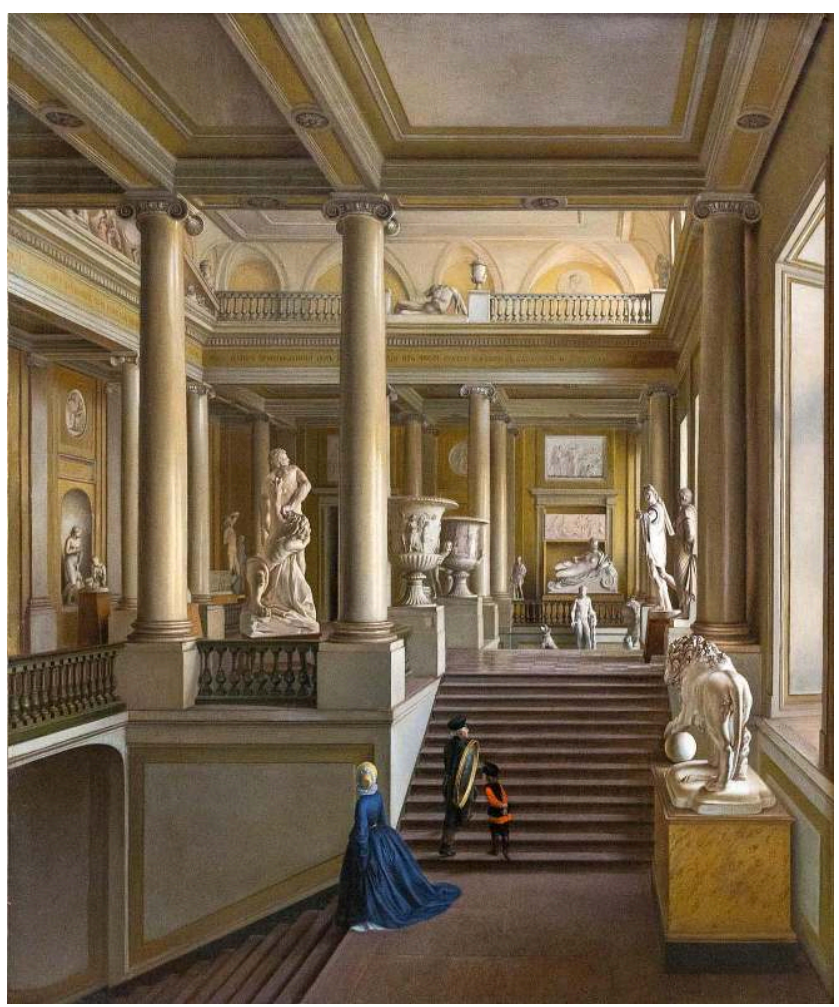

L'Escalier principal de l'Académie des Arts, Ivan Alekseevich Ivanov (1779-1848), 1830, aquarelle (106 x $88 \mathrm{~cm}$ ) conservée au Musée de la recherche à l'Académie russe des arts de Saint-Pétersbourg Reproduction Académie des beaux-arts de Saint-Pétersbourg.

Sur les bords de la Neva, en face des célèbres sphinx égyptiens qui trônent sur le quai de l'île Vassilievski, le «musée de la Recherche scientifique de l'Académie des beauxarts " présente aujourd'hui différentes écoles européennes - italiennes, allemandes, flamandes, françaises, belges, $\mathrm{du} \mathrm{XV}^{\mathrm{e}}$ au $\mathrm{xIX}^{\mathrm{e}}$ siècle - comprenant notamment des 
œuvres de Luca Giordano, Pier Francesco Mola (1612-1666), Angelika Kauffmann (1841-1807), Annibale Carracci (1560-1609), Le Guerchin, Abraham Hondius (1625, 1631 ?-1691), Anton Raphael Mengs, etc. Pourtant, il ne reste qu'une dizaine de tableaux de la collection de peinture de Shuvalov initialement léguée à l'Académie en 1758.

Figure 9

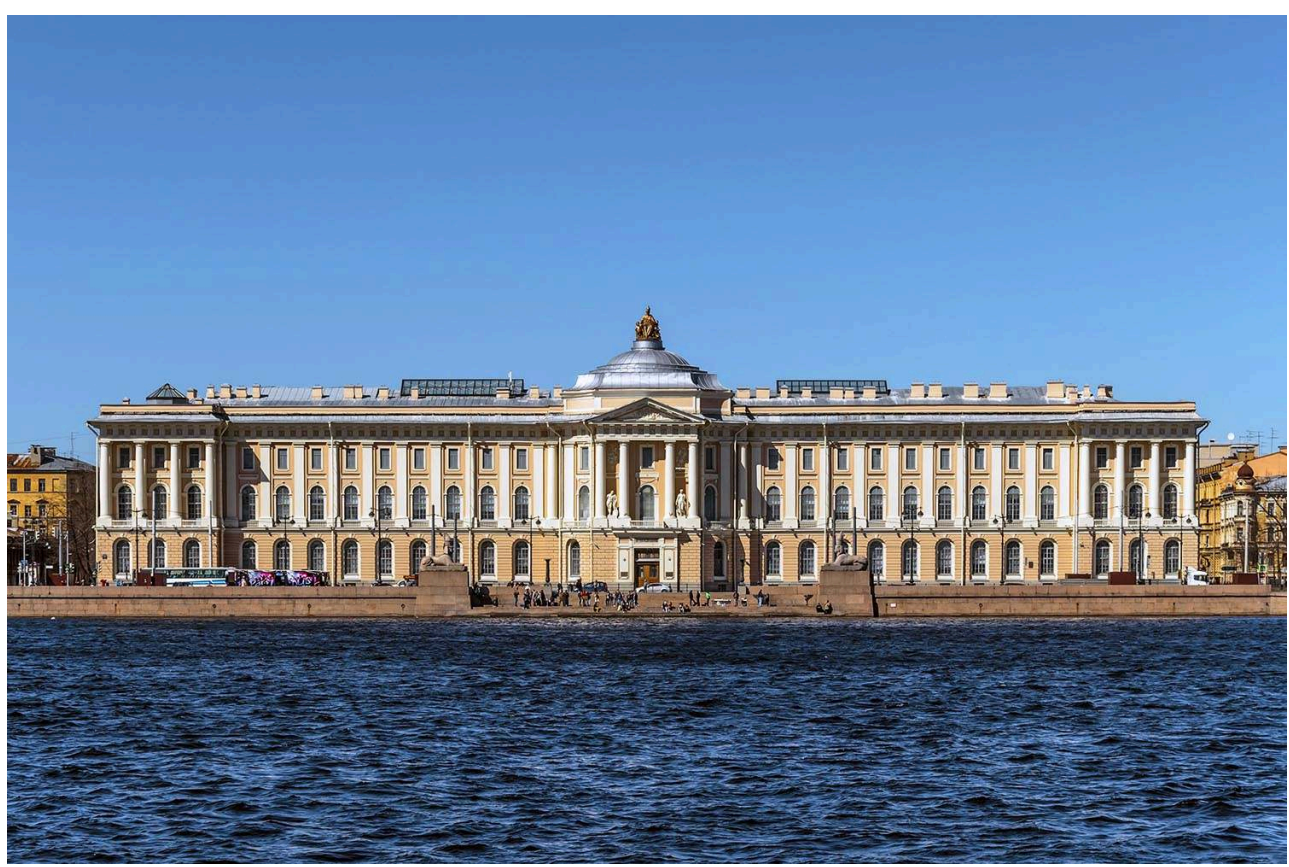

Académie des Beaux-Arts de Saint-Pétersbourg, 2013.

(c) Photo Alex Fedorov / reproduction Wikimedia Commons.

19 À la fin du premier quart $\mathrm{du} \mathrm{XIX}^{\mathrm{e}}$ siècle, la collection de tableaux de l'Académie impériale des arts, en valeur et en nombre de tableaux, n'était que légèrement inférieure à celle de l'Ermitage. Les collections académiques ont en effet été transférées à plusieurs reprises dans d'autres musées, d'abord au moment de la création d'un Musée russe par Nicolas $\mathrm{I}^{\mathrm{er}}$ - qui fait réaménager les salles d'exposition de l'Académie en les remplissant de plâtres d'antiques - puis lorsque l'Académie elle-même est supprimée en 1917. Les œuvres les plus précieuses de la collection académique sont alors transférées au musée de l'Ermitage (1918), notamment celles de la donation Shuvalov, ainsi que celle du comte Gregorij Alexandrovic Kushelev-Bezborodko, qui en 1863 avait doté le musée d'une vaste collection de peintures et de sculptures.

La collection historique a ainsi été largement dispersée, et seules les collections de plâtres sont demeurées cohérentes. Une importante collection de dessins pédagogiques académiques du XVIII ${ }^{\mathrm{e}}$ au $\mathrm{XX}^{\mathrm{e}}$ siècle en Russie demeure ${ }^{11}$ cependant, constituée d'études sur la bosse ou le modèle vivant réalisées par des étudiants de l'Académie, de copies ou de compositions originales, permettant de retracer en détail l'évolution de la pédagogie jusqu'à nos jours, car l'institution a mis en œuvre une vraie politique de dépôt de dessins de ses étudiants et de ses professeurs.

21 Les salles d'exposition permanente du musée se déploient aujourd'hui sur plusieurs étages. Le premier est occupé par les plâtres d'antiques ${ }^{12}$ et d'ornements 
architecturaux, dont un grand nombre ont été moulés à partir des originaux des XVIII et XIX ${ }^{e}$ siècles [fig. 10]. Les salles du deuxième étage présentent une partie des peintures de la collection historique du musée, qui ont servi de modèles pour l'étude à des générations de jeunes Russes. Enfin, le troisième étage est consacré aux dessins et maquettes des chefs-d'œuvre architecturaux de Saint-Pétersbourg, y compris des projets non réalisés. Il s'agit de l'étage le plus apprécié du musée car il n'existe aucun autre endroit à Saint-Pétersbourg consacré à l'urbanisme de la ville.

Figure 10

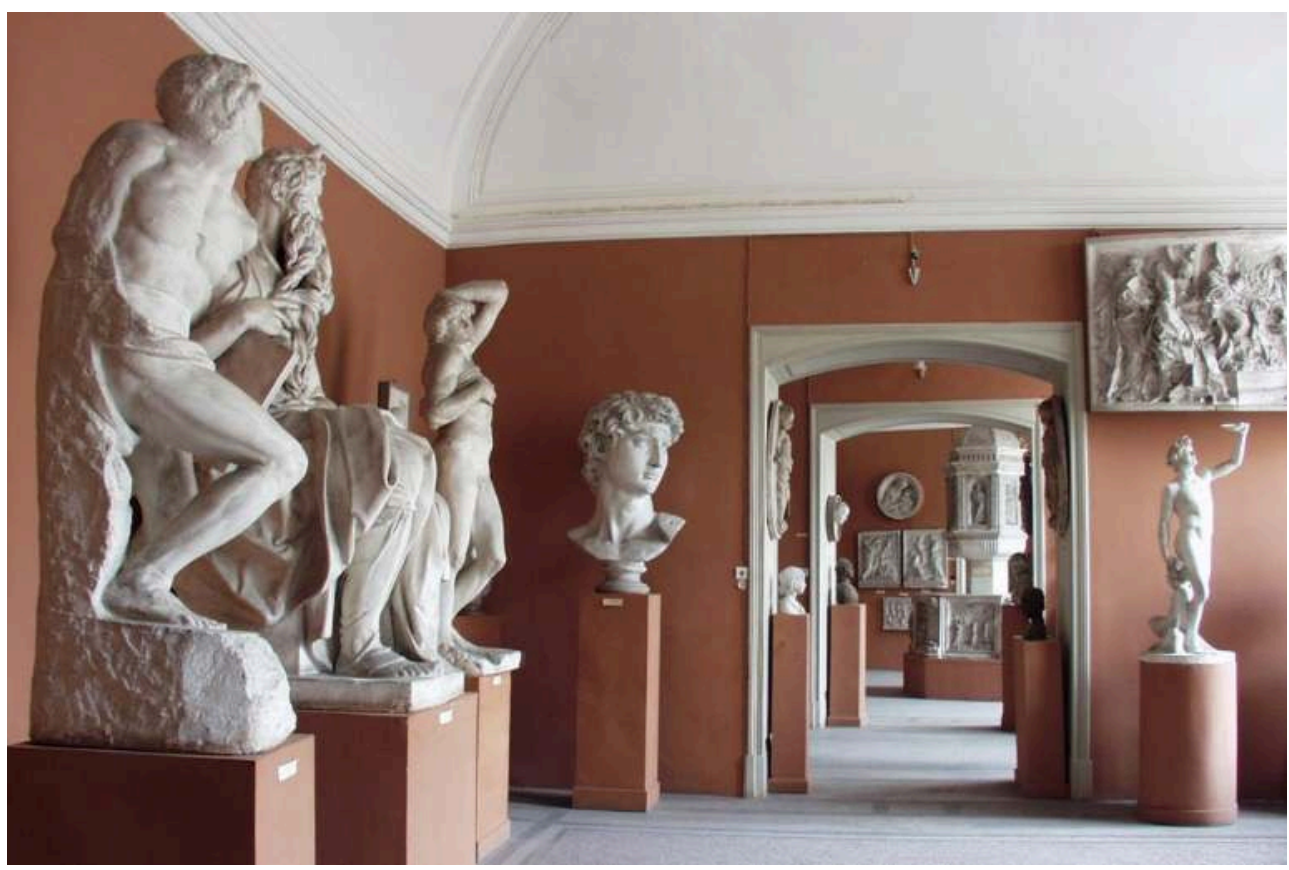

Vue d'une salle du rez-de-chaussée du musée de l'Académie des beaux-arts de Saint-Pétersbourg (c) Photographe inconnu (Académie des Beaux-Arts de Saint Pétersbourg).

Quoique le parcours permanent ne bénéficie pas toujours d'une muséographie moderne, ce dernier a le mérite de montrer combien la culture esthétique de SaintPétersbourg diffuse, à travers l'Académie, les principes du néoclassicisme, sous l'égide de l'architecte d'origine allemande Georg Friedrich Veldten, directeur de l'Académie de 1789 à 1801 . Alors qu'à Moscou les étudiants sont incités à peindre d'après nature, cette pratique est absente de l'enseignement pétersbourgeois ${ }^{13}$. Les deux principales villes du pays se distinguent à la fin $\mathrm{du}_{\mathrm{XIX}} \mathrm{e}^{\mathrm{e}}$ siècle et au tout début $\mathrm{du} \mathrm{xx}^{\mathrm{e}}$ siècle par des systèmes d'enseignement distincts, ce que les collections exposées montrent parfaitement. L'exposition permanente passe en effet assez brutalement du néoclassicisme aux travaux académiques et les diplômes exécutés dans les années 1920-1930, passant sous silence la contestation de jeunes artistes russes qui, au milieu du xix siècle, revendiquent la liberté de peindre des sujets réalistes et rejettent le style imposé par Jean-Auguste-Dominique Ingres qui est alors pris comme modèle à l'Académie. Ce mouvement, dit des "Ambulants », s'est publiquement détaché de l'Académie en 1863, préférant exposer de manière itinérante à travers la Russie. Sans transition, une salle d'exposition permanente offre une quantité d'œuvres des années 1926-1932, témoignant d'une période de recherches et d'expérimentations plastiques qui s'achève au début des années 1930, avec le décret du Comité central du Parti communiste «sur 
la restructuration des organisations littéraires et artistiques » (1932) et la proclamation $\mathrm{du}$ « réalisme socialiste » comme style unique ${ }^{14}$.

Si la mise en valeur muséale des collections de ces trois institutions est dans l'ensemble appréciée, les collections ont une audience confidentielle, souvent exposées à l'écart des grands circuits muséaux. Le plus problématique est certainement qu'il n'existe aucun discours sur l'ensemble de la collection et de son histoire, ni sur l'histoire de la formation artistique, soit que certains chefs-d'œuvre absorbent par trop la visibilité de l'ensemble, dans le cas de Madrid et de Vienne, soit que la collection soit trop lacunaire au regard de son fonds historique comme à Saint-Pétersbourg. Si la question de la formation des artistes et des modèles proposés est présente de manière implicite, elle ne constitue pas la colonne vertébrale de la muséographie. Les visiteurs ont dans l'ensemble l'impression de visiter un musée de "second ordre ", peu lisible dans ses objectifs.

\section{En Italie et en Allemagne : une césure opérée entre l'art ancien et l'art contemporain}

Le cas des académies italiennes, qui se séparent de toutes de leurs galeries d'art au lendemain des réflexions engendrées par l'Unité du royaume d'Italie (1861), n'est que brièvement évoquée ici, faisant l'objet d'un article spécifique dans ce numéro ${ }^{15}$. De manière générale, la situation des galeries qui dépendent des académies des beaux-arts est jugée problématique du point de vue de leur gestion dès les années 1860 . Après de nombreux débats liés aux relations historiques et structurelles entre écoles et galeries muséales, la conservation et l'exposition des œuvres d'art anciennes est jugée peu compatible avec le fonctionnement d'une école d'art. En 1882, un décret royal soustrait donc la gestion des galeries d'art historiques aux académies, qui deviennent des institutions autonomes placées sous la tutelle du ministère de l'Éducation.

À l'image des écoles italiennes, les écoles d'art allemandes ont essaimé sur tout le territoire à des dates très précoces, de la fin du Xvir siècle jusqu'au milieu du XVIII ${ }^{e}$ siècle. Dans les régions germanophones, comme dans l'Italie de la Renaissance, des écoles de dessin informelles sont apparues avant de devenir des académies souvent fondées sur le modèle parisien ${ }^{16}$. Le polycentrisme artistique propre à l'Allemagne, et sa structure politique en Länder a maintenu cette originalité et cette richesse.

Les académies allemandes, créées sous l'impulsion des artistes ${ }^{17}$, ont été conçues avant tout comme des institutions d'enseignement et de formation. Aucune collection muséale n'a été rassemblée, les grands aristocrates collectionneurs ouvrant par ailleurs volontiers au public leurs collections. Les académies d'art allemandes ont ainsi constitué principalement des collections pédagogiques, en conservant les œuvres d'élèves ou de professeurs avec une politique de dépôt variable selon les directorats. Les fonctions muséales et pédagogiques des écoles d'art ont été très naturellement distinguées et ces écoles se consacrent aujourd'hui exclusivement à la formation.

En effet, les artistes en formation dessinent d'après l'antique en dehors de l'académie, et la distinction entre collection privée et collection publique, vocation pédagogique et historique, n'est pas pertinente au XvIII ${ }^{\mathrm{e}}$ siècle d'une manière générale ${ }^{18}$ et plus particulièrement en territoire germanophone, où l'ouverture de galeries privées sous la forme de musées indépendants est très précoce au regard des pays européens voisins ${ }^{19}$. 
C'est pour cette raison qu'à Dresde, lorsqu'une partie des plâtres d'antiques du peintre Anton Raphaël Mengs - 800 pièces qui ne faisaient pas partie du legs madrilène - fut acquise par le roi de Saxe Frédéric-Auguste pour son académie d'art, ce dernier choisit d'emblée de l'exposer dans un musée public ${ }^{20}$ et non de la réserver à la seule académie.

Le territoire allemand, et c'est un fait majeur, est par ailleurs très concerné par la constitution de collections publiques de plâtres d'antiques, en raison de l'implication d'archéologues, d'historiens d'art et d'artistes allemands dans l'étude de l'art antique. La personnalité de l'historien de l'art prussien Johann Joachim Winckelmann, auteur des Réflexions sur l'imitation des ceuvres grecques dans la sculpture et la peinture (1755) puis d'une Histoire de l'art chez les Anciens (1764), ouvrages qui dessinent les bases théoriques de ce que sera le mouvement néoclassique européen, y tient une place majeure. Ses idées sont notamment relayées par le peintre Anton Raphaël Mengs, né en Bohême, élevé à Dresde et établi à Rome. Nombreux sont les artistes défenseurs de la doctrine néoclassique qui se retrouvent directeurs d'académies, comme le peintre et sculpteur Johann Gottfried Schadow, proche du sculpteur Antonio Canova, nommé en 1815 directeur de l'Académie de Berlin.

Cependant, en raison du succès de la science antiquaire, ce sont parfois les universités et non les académies d'art qui ont constitué des collections de plâtres d'antiques, comme à Göttingen ${ }^{21}$ en 1767 et à Bonn en $1819^{22}$, avant même la création de chaires d'archéologie classique ou d'histoire de l'art. Parfois, les universités ont pu récupérer les collections initialement destinées à une académie, comme dans le cas de Mannheim. Sa fameuse galerie des plâtres antiques, transférée à l'Académie de Münich en 1807, est aujourd'hui conservée et exposée par les soins de l'université [fig.11]. Le nombre de gypsothèques universitaires ouvertes au public, à la muséographie plus ou moins élaborée, est impressionnant, comme le montre le portail récemment créé consacré aux collections de plâtres en Allemagne ${ }^{23}$ : chaque Land d'Allemagne possède sa gypsothèque, qu'elle dépende d'une institution muséale ou d'une université. 
Figure 11

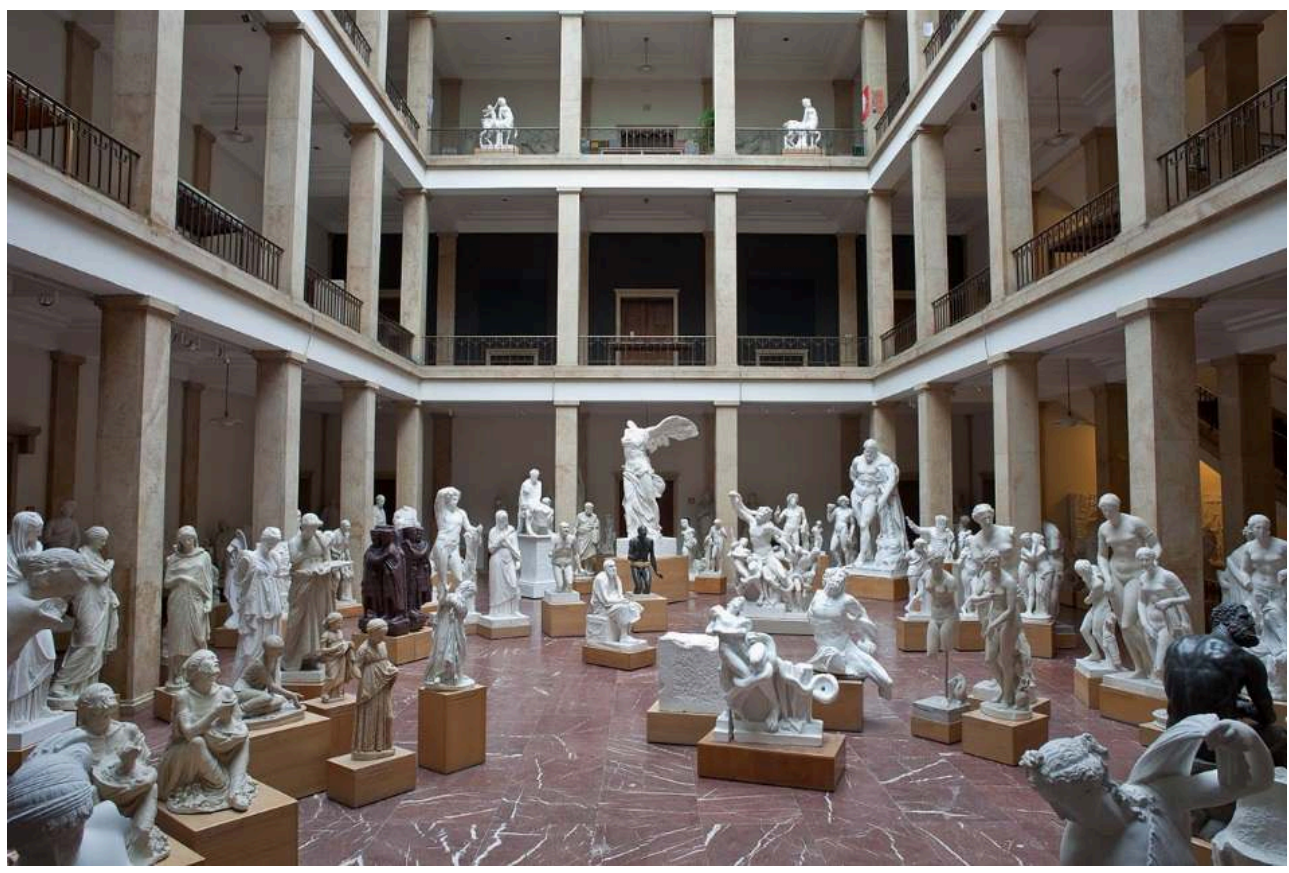

Vue d'une salle du musée des Moulages classiques à Münich.

(c) Photographe inconnu (Museum für Abgüsse Klassischer Bildwerke München).

30 Les écoles d'art ont par ailleurs été profondément transformées après la Seconde Guerre mondiale. Des fusions ont eu lieu, souvent pour des raisons financières, avec d'autres structures impliquant l'artisanat d'art ou un enseignement technique. Dans le cas de Berlin, il n'existe pas forcément de coordination entre les quatre zones d'occupation (américaine, britannique, française et soviétique) et dans d'autres villes, les choix ont parfois été faits en raison d'une reconstruction parfois difficile ${ }^{24}$. Le patrimoine conservé aujourd'hui au sein des écoles d'art est essentiellement graphique et dans certains cas, déposé dans le musée de la ville. Ainsi, à Düsseldorf, la fameuse collection de dessins du peintre Lambert Krahe (1712-1790) ${ }^{25}$ qui fonde l'Académie des arts en 1762, est aujourd'hui conservée au Kunstpalast. Quelques exemples seront évoqués afin de donner un aperçu, non exhaustif, de la manière dont est abordé le patrimoine des écoles d'art en Allemagne.

31 L'Académie des arts de Berlin, fondée en 1696, sise sur la Pariser Platz (la "place de Paris »), près de la porte de Brandebourg, conserve aujourd'hui une collection avant tout graphique, comprenant environ 60000 œuvres sur papier - dessins ${ }^{26}$, peintures sur papier, gravures, photographies artistiques ainsi que plus de 50000 affiches d'artistes. L'Académie mène une active politique de recherche et de numérisation de ses archives $^{27}$ ainsi que de son fonds graphique, en partenariat avec l'université. Son fonds d'archives retient particulièrement l'attention, étant le plus important dans le domaine de l'art et de la culture moderne dans les pays germanophones ${ }^{28}$. L'Académie est également davantage tournée vers la question des acquisitions et de la constitution de collections contemporaines. Pour exemple, à l'initiative de Hans-Joachim Burgert (1928-2009), l'un des plus importants calligraphes allemands, l'institution a pu constituer une collection de calligraphies en suscitant de nombreux dons. Parmi ses acquisitions récentes figurent la plus grande collection d'œuvres sur papier de l'artiste est-allemand Joachim John (1933-2018) et de Dieter Goltzsche (1934-), l'œuvre 
graphique du suisse Alfonso Hüppi (1935-), les photogrammes expérimentaux de Heinz Hajek-Halke (1898-1983) ainsi qu'une vaste série de tirages de Michael Ruetz (1940-).

La seule collection de plâtres d'antiques donne une idée du destin complexe des collections patrimoniales académiques. L'Académie de Berlin avait constitué dès sa fondation une collection de plâtres d'antiques, transférée en 1855 au premier étage et du Neues Museum sur l'île aux musées de Berlin. À partir de 1921, la collection est exposée dans les nouveaux locaux de l'Université de Berlin, aujourd'hui Université Humboldt. Avec environ 2500 plâtres, il s'agissait de l'une des plus grandes collections au monde, dont une partie a été détruite pendant la Seconde Guerre mondiale. Enfin, à l'ouest de Berlin, une collection de plâtres d'antiques a été reconstruite au sein de l'Université libre ${ }^{29}$, en coopération avec le Staatliche Museen zu Berlin et a ouvert ses portes en 1988 à Charlottenbourg ${ }^{30}$, tandis qu'une partie de l'ancienne collection a été rendue accessible à l'Université Humboldt après 1990.

En revanche, le patrimoine académique, lorsqu'il est spécifique à l'enseignement, a été conservé in situ. L'école des Beaux-Arts de Dresde (Kunstakademie Dresden), fondée en 1764, l'une des plus anciennes écoles d'art d'Allemagne, possède en effet un cabinet d'anatomie à destination des artistes sans équivalent sur le territoire ${ }^{31}$. Ce cabinet est aujourd'hui au centre des intérêts de l'école par le biais d'un projet européen consacré au patrimoine des écoles d'art en Europe. La collection anatomique de l'Académie des beaux-arts de Dresde est, avec les collections des Académies d'art de Paris et de SaintPétersbourg, l'un des derniers témoignages de l'intérêt marqué pour l'anatomie au sein de la formation artistique. Cette collection est composée d'environ 600 objets et plus d'un millier de supports pédagogiques datant du début du XIX siècle aux années 2000. En 1766, le premier squelette en os a été acquis en 1766, alors que les cours d'anatomie sont donnés par le peintre Giovanni Battista Casanova (1730-1795). Ce patrimoine est particulièrement intéressant sur le territoire allemand si influencé par le néoclassicisme, dans la mesure où il apparaît bien comme la discrète survivance du modèle naturaliste qui avait dominé l'esthétique allemande avec Dürer et l'école flamande.

\section{La Royal Academy de Londres : une exposition permanente capable de renforcer l'identité culturelle de «l'école anglaise »}

La fondation de la première académie des beaux-arts en Angleterre [fig. 12] est relativement tardive (1768) et a donné naissance à un modèle académique tout à fait spécifique, puisque les artistes ont pu faire la démonstration que le revenu des expositions pouvait assurer une indépendance financière, donc politique, inconnue des Académies du reste de l'Europe. Le pragmatisme qui caractérise l'approche anglosaxonne des problèmes ainsi que leur peu de goût pour les débats théoriques se retrouvent clairement dans les collections aujourd'hui conservées : les références sont plurielles et le modèle gréco-romain n'y domine $\operatorname{pas}^{32}$. L'institution est marquée du sceau de l'indépendance; exclusivement dirigée par des artistes, elle veut s'attacher à concilier les idéaux de l'art et la demande du marché. L'institution parvient même à intégrer le médecin William Hunter (1718-1783), ou des personnalités aussi singulières que le sculpteur John Flaxman (1755-1826), l'architecte John Soane (1753-1837), les peintres John Constable (1776-1837) ou J. M. W. Turner (1775-1851). Sa collection est 
majoritairement constituée d'œuvres d'artistes membres, aujourd'hui riche de près de 900 tableaux (Joshua Reynolds, Thomas Gainsborough, John Constable ou David Hockney), plus de 300 sculptures, 25000 dessins, gravures et photographies, et plus de 500 plâtres d'après l'antique, sans compter les médailles et livres précieux. La collection témoigne de l'éclosion d'une école anglaise de peinture qui s'est ainsi faite à la faveur d'un rapprochement inédit entre art et nation à travers la Royal Academy of Arts ${ }^{33}$, avec ses genres de prédilection dans le domaine de la peinture, le portrait et le paysage.

Figure 12

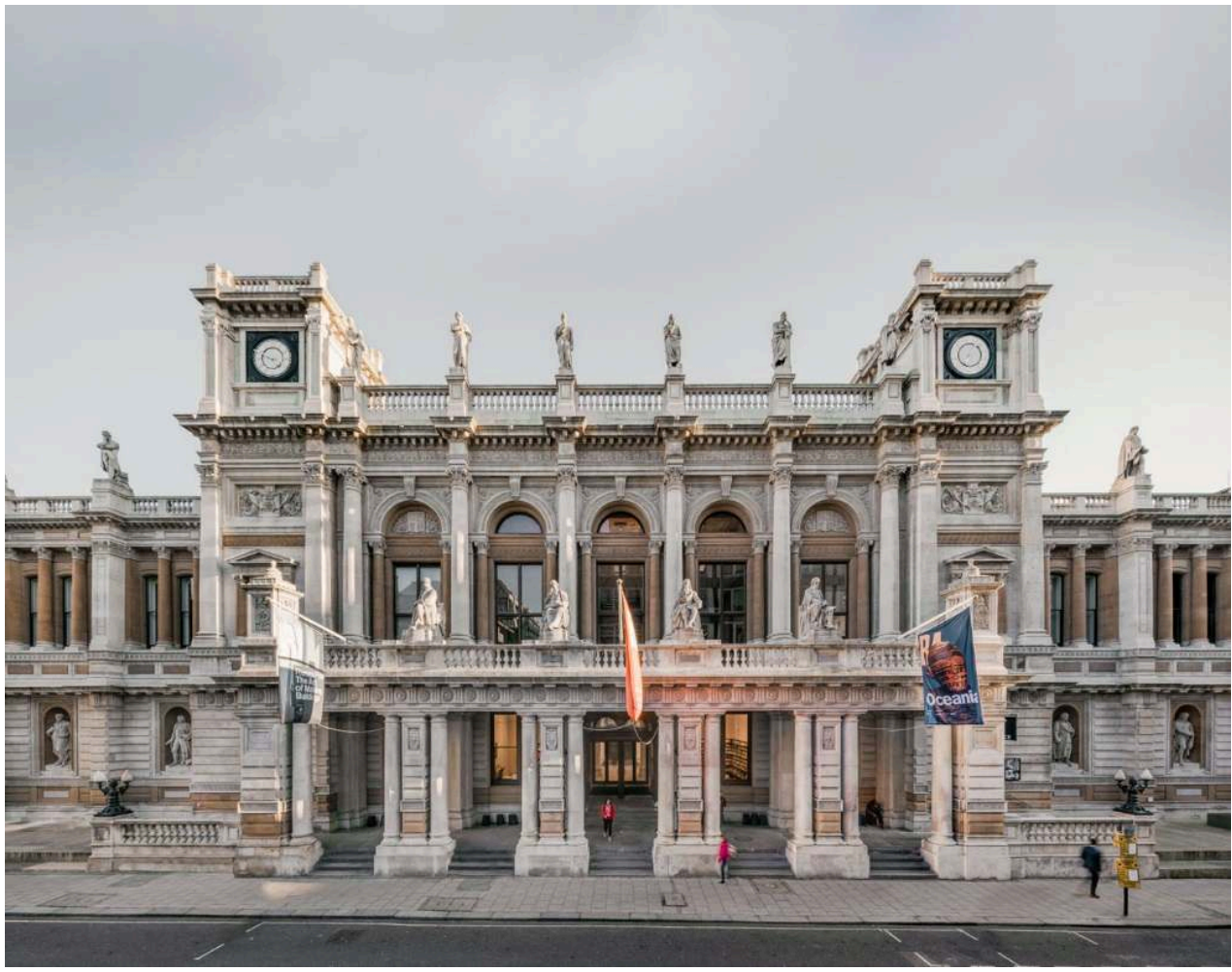

Royal Academy de Londres, 2018.

(c) Photo Simon Menges.

À l'occasion des deux cent cinquante ans de l'institution, en mars 2018, la Royal Academy a inauguré son extension conçue par l'architecte David Chipperfield, laquelle a permis d'exposer de manière permanente une partie des collections de l'école, avec le commissariat de Christopher Le Brun, ancien professeur de dessin et directeur de l'institution [fig. 13, fig. 14]. Au sein d'une galerie souterraine qui relie les deux bâtiments de l'école, un parcours a été pensé, qui monte jusqu'au premier étage de Burlington Gardens. Au total, six pièces et un petit corridor permettent à la Royal Academy de présenter une partie de sa collection, pédagogique et historique. L'idée est d'expliquer comment le dessin était enseigné au cours de l'histoire, en confrontant par exemple le plâtre de l'Hercule Farnèse, venu d'Italie en 1790, aux divers modèles anatomiques produits à la fin du XVIII ${ }^{\mathrm{e}}$ siècle et au début du XIX ${ }^{\mathrm{e}}$, dont le moulage d'un condamné à mort exécuté en 1801, posé en crucifié et offert en objet de débats sur les incorrections anatomiques des images du Christ sur la croix. Au-delà de l'anecdote, la muséographie montre par-là l'indépendance des artistes à l'égard du modèle antique en ce qui concerne le dessin de la figure et l'importance spécifiquement anglaise de 
l'observation dans le processus de création artistique, fruit d'une longue tradition philosophique et littéraire qui prône la découverte et l'observation attentive des phénomènes de la nature, $\mathrm{y}$ compris la nature humaine ${ }^{34}$.

Figure 13

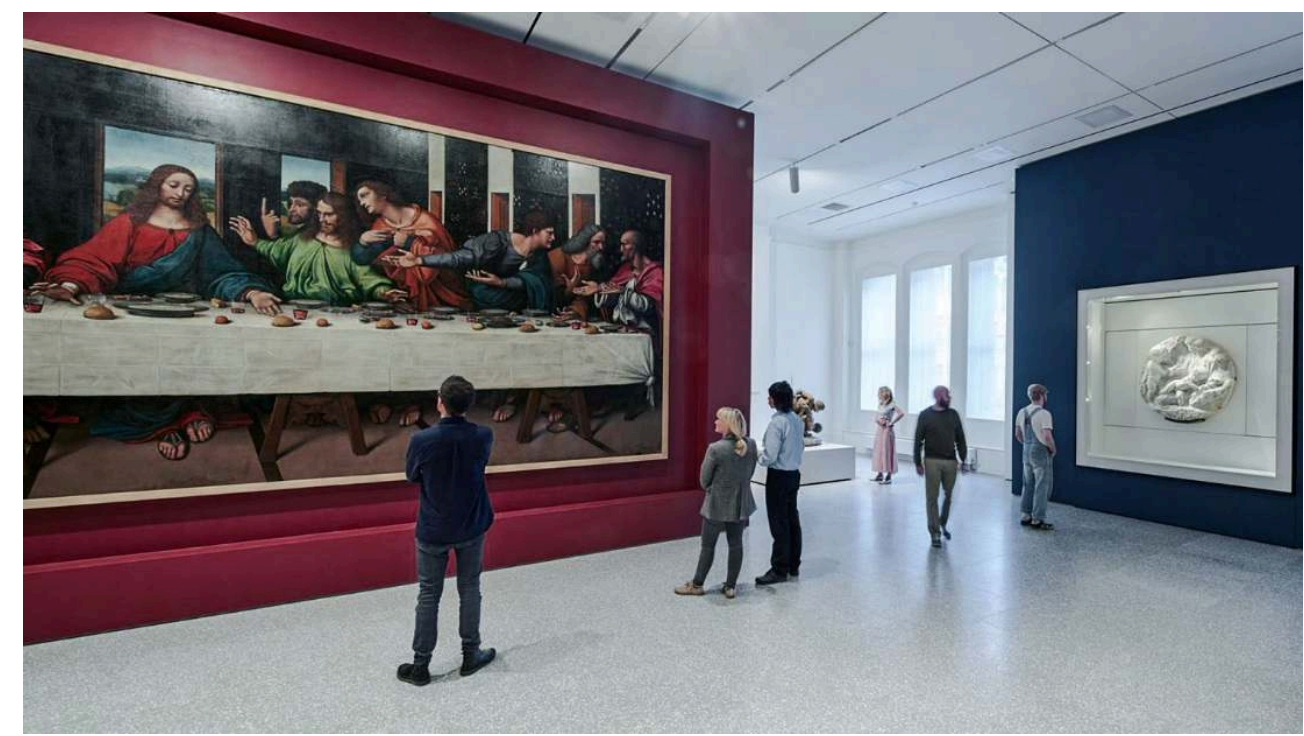

Vue d'une salle d'exposition présentant une copie de La Cène de Léonard de Vinci et la Vierge à l'Enfant avec saint Jean-Baptiste, dit Tondo Taddei, de Michel-Ange à la Royal Academy of Arts de Londres, 2018.

(c) Photo Simon Menges.

Figure 14

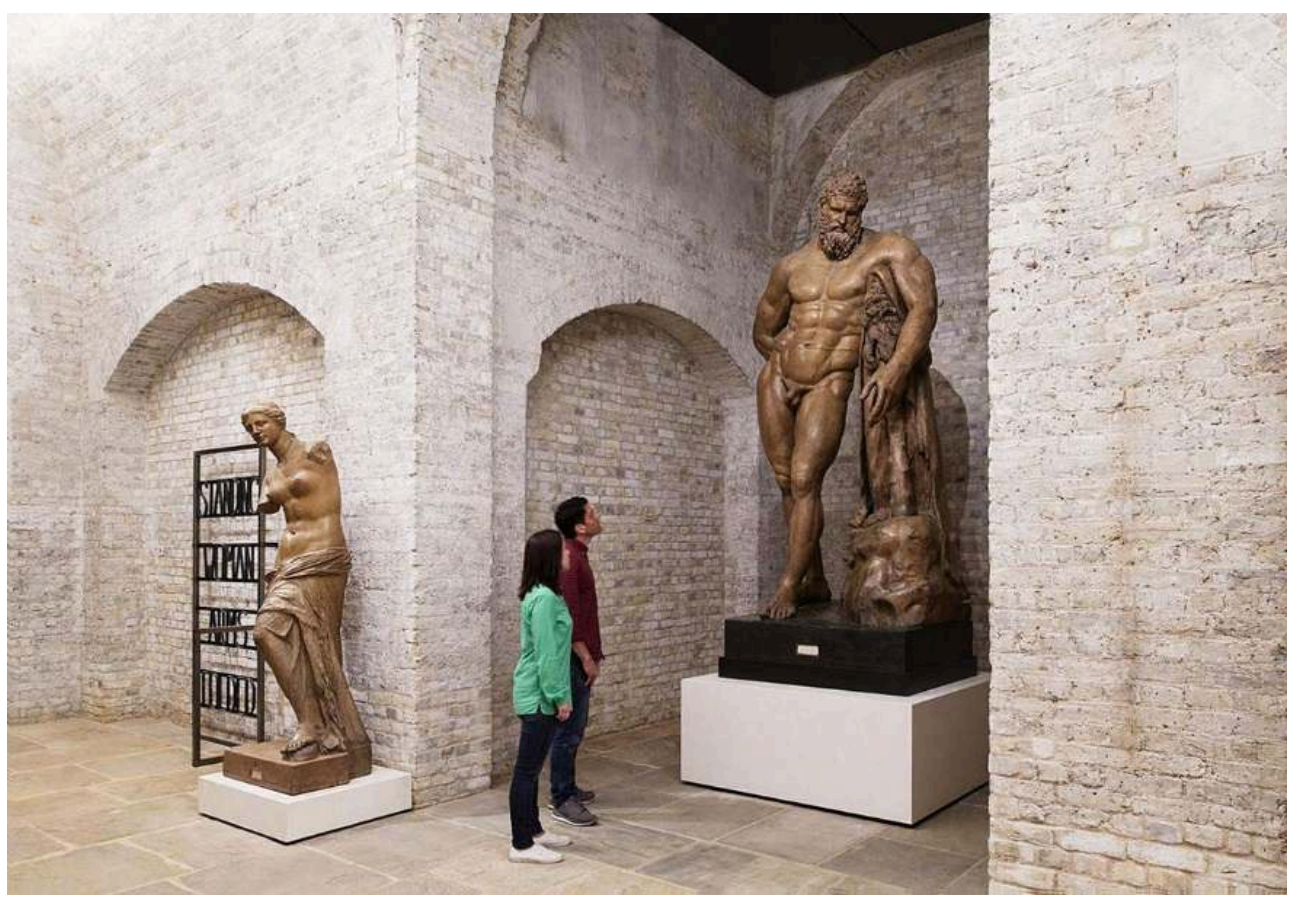

Vue d'une galerie d'exposition présentant sculptures et plâtres d'antique à la Royal Academy of Arts de Londres, 2018.

(c) Photo James Harris. 
Ce sont bien des modèles aux esthétiques parfois opposées qui forment le fonds des collections de la Royal Academy ${ }^{35}$. On y trouve aussi bien le tondo en marbre de MichelAnge qu'une copie de la Cène de Léonard de Vinci. En face se trouve la copie de trois cartons de tapisserie de Raphaël, exécutés au début du xvIII siècle par James Thornhill, ainsi qu'une cimaise exposant les dessins anciens inspirés par le Jugement dernier de Michel-Ange. Christopher Le Brun a exhumé des caves l'immense Satan rassemblant ses légions [fig. 15] de Thomas Lawrence (1797), majestueuse démonstration de la veine fantastique anglaise, aux côtés du «cœur» plus traditionnel de l'école britannique, avec les paysages de Thomas Gainsborough et les portraits des plus illustres membres de l'Académie, dont Joshua Reynolds. Un vaste corridor permet enfin de présenter nombre de plâtres ornementaux. La démonstration historique et esthétique subtile du parcours permanent, donnant à voir la variété des modèles plastiques, s'accompagne d'expositions contemporaines ou de travaux d'étudiants, voire d'académiciens comme Anish Kapoor (1954-) qui, en 2009, est devenu le premier artiste vivant à avoir droit à une exposition personnelle occupant tout le palais.

Figure 15

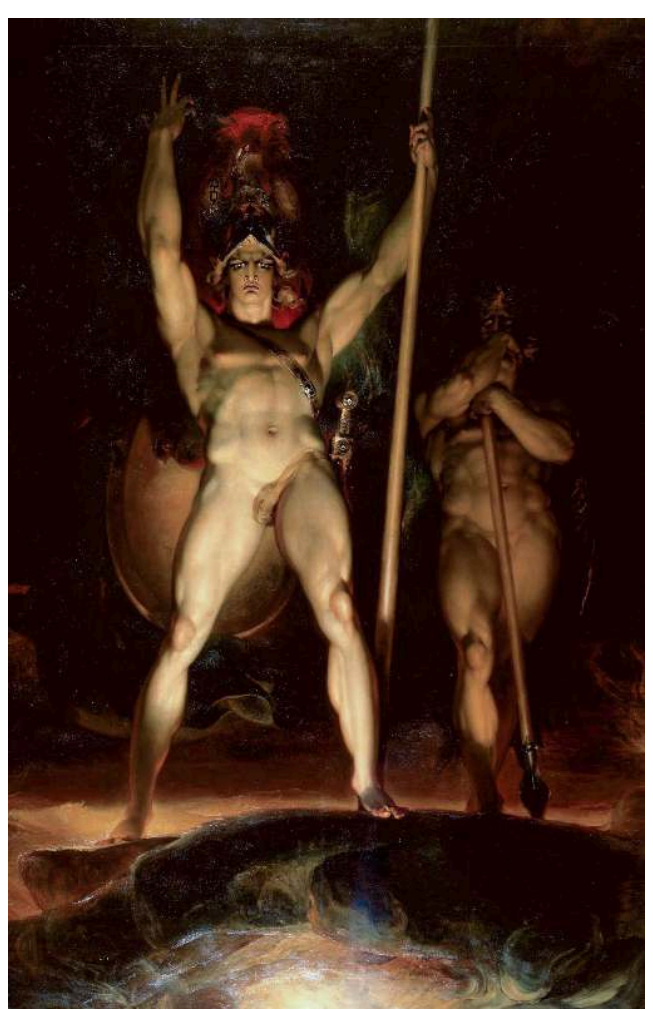

Satan summoning his Legions, Sir Thomas Lawrence (1769-1830), 1796-1797, huile sur toile (431 x $274 \mathrm{~cm}$ ) conservée à la Royal Academy of Arts (inv. 03/1094), Londres.

Reproduction Royal Academy of arts.

Avec ce parcours permanent historique, la Royal Academy renoue avec le principe qu'elle avait institué depuis 1870, en proposant en hiver, à côté de l'exposition d'artistes vivants [fig. 16] ${ }^{36}$, une exposition consacrée aux maîtres anciens. Mais si la collection de l'école, qui compte près de 46000 œuvres, est mise à l'honneur, la Royal Academy se conçoit avant tout comme une école doublée d'un centre d'expositions et non comme un musée. 
Figure 16

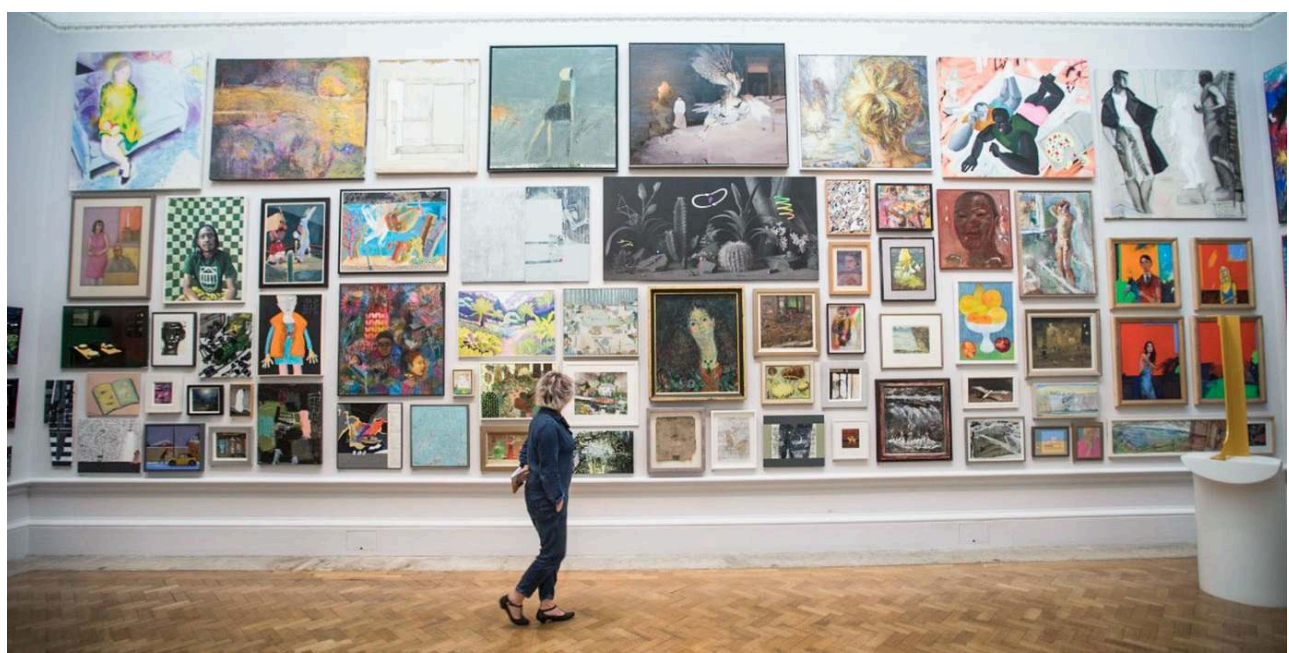

Exposition des travaux d'étudiants (« Royal Academy Summer Exhibition ») au sein du parcours permanent à la Royal Academy of Arts, Londres, 2018.

(c) Photographe inconnu (Royal Academy of Arts).

Le panorama du patrimoine européen des écoles d'art ici esquissé montre indéniablement qu'il suscite aujourd'hui l'intérêt, quels que soient les partis pris choisis, à savoir une exposition permanente ou une simple conservation érudite au sein d'une institution pédagogique ou muséale. L'existence de musées bien identifiés dans le giron de certaines écoles, comme à Madrid, Vienne ou Saint-Pétersbourg contribue certainement, à l'heure où le succès des institutions patrimoniales au sens large ne se dément pas, à repenser au sens de l'exposition des collections académiques, car ces dernières témoignent de l'histoire du regard porté sur les modèles italiens, puis l'art gréco-romain ou la ténacité du modèle naturaliste. C'est ainsi une histoire du regard de l'artiste en formation, mais aussi d'une nation, qui se révèle, comme le montrent les choix opérés à la Royal Academy de Londres, qui expose de manière permanente une partie de ses collections au sein d'un projet de rénovation global du bâtiment.

\section{NOTES}

1. WEBER Carmen Sylvia (dir.), Verborgene Schätze aus Wien. Meisterwerke von Dürer, Botticelli, Rembrandt, Rubens, Amerling, Klimt und Hundertwasser u.a. Die Kunstsammlungen der Akademie der bildenden Künste Wien zu Gast in der Kunsthalle Würth, Künzelsau, Swiridoff Verlag, 2017.

2. FLEISCHER Martina, HAGEN Bettina, KOCH Claudia et al., La Galerie de peinture de l'Académie des beaux-arts de Vienne, Londres, Scala, 2005, p. 9.

3. PEZZL Johann, Nouvelle description de Vienne, capitale de l'Autriche..., Vienne, G. de Moesle, 1818, p. 233.

4. Guide des étrangers à Vienne, Vienne, Mörschner et Jasper, 1838, p. 180. 
5. BÉDAT Claude, L'Académie des beaux-arts de Madrid (1744-1808). Contribution à l'étude des influences stylistiques et de la mentalité artistique de l'Espagne au XVIII e siècle, Toulouse, Association des publications de l'université de Toulouse-Le Mirail, coll. «Publications de l'université de Toulouse-Le Mirail », série « A », 1973.

6. GÉAL Pierre, La Naissance des musées d'art en Espagne. XVIII ${ }^{e}-X I X^{e}$ siècles, Madrid, Casa de Velázquez, coll. «Bibliothèque de la Casa de Velázquez », 2005, p. 38.

7. MARTÍNEZ FRIERA Joaquin, Un Museo de Pinturas en el Palacio de Buenavista. Proyecto de la Real Academia de Nobles Artes de San Fernando, Madrid, Afrodisio Aguado, 1942, p. 40-45.

8. GÉAL Pierre, La Naissance..., p. 187.

9. L'Académie acquiert au XVIII ${ }^{\mathrm{e}}$ siècle la fameuse collection de dessins laissée par le peintre Carlo Maratti (1625-1713), comptant plus de 1000 feuilles de ce maître. Au siècle suivant, des volumes contenant 500 dessins environ, pour la plupart d'artistes italiens des $\mathrm{XVI}^{\mathrm{e}}$ et $\mathrm{XVII}^{\mathrm{e}}$ siècles, provenant du monastère de Valparaiso en Zamora. Voir GERARD POWELL Véronique \& MENA MARQUÉS Manuela, «Les collections de dessins italiens en Espagne », Revue de l'art, 1985, nº 70, p. 83-89 [disponible en ligne], https://www.persee.fr/doc/ rvart_0035-1326_1985_num_70_1_347538 [lien valide en novembre 2020].

10. BAUDEZ Basile, «Du Moujik à l'artiste. L'Académie impériale des beaux-arts de SaintPétersbourg (1757-1802) », thèse de doctorat en histoire moderne soutenue à l'École nationale des chartes (Paris, 2000).

11. S. N., Anniversary Directory graduates of Saint Petersburg State Academic Institute of Painting, Sculpture, and Architecture named after Ilya Repin, Russian Academy of Arts. 1915-2005I, SaintPétersbourg, Pervotsvet, 2007.

12. La collection comprend au total près de 3000 plâtres : sculptures antiques et européennes du $\mathrm{XII}^{\mathrm{e}}$ au XIX ${ }^{\mathrm{e}}$ siècles, mais aussi du Proche-Orient, ainsi que des plâtres ornementaux russes.

13. MILBACH Juliette, «L'enseignement artistique en Russie dans les années 1910 : la pédagogie moscovite en trois étapes ", Revue des études slaves [en ligne], $\mathrm{n}^{\circ}$ 87, vol. 3-4, 2016, https:// journals.openedition.org/res/pdf/946 [lien valide en novembre 2020].

14. PISHNY Igor N., The Leningrad School of Painting. Socialist Realism of 1930-1980s, SaintPétersbourg, Kolomenskaya Versta, 2008.

15. Voir l'article de Martine Lerda dans le présent numéro.

16. La première académie allemande est fondée vers 1674 à Nuremberg, puis à Berlin, en 1696, Augsbourg en 1710, Düsseldorf en 1762, Dresde en 1764 et Karlsruhe en 1770.

17. Peter Anton von Verschaffelt à Mannheim, Lambert Krahe à Düsseldorf, les frères Terwesten à Berlin, Roman Anton Boos et de Johann Christian von Mannlich à Munich, ou Christian Ludwig von Hagedorn à Dresde.

18. HASKELL Francis \& PENNY Nicholas, Pour l'amour de l'Antique. La statuaire gréco-romaine et le goût européen, 1500-1900, Paris, Hachette, coll. « Pluriel », 1999 [1981], p. 123.

19. SAVOY Bénédicte, “ “Comme dans un ciel étoilé.” Éléments pour une géographie culturelle de l'Allemagne ", in ALLARD Sébastien \& COHN Danièle (dir.), De l'Allemagne, 1800-1939. De Friedrich à Beckmann, catalogue d'exposition (Paris, musée du Louvre, 28 mars-24 juin 2013), Paris, Hazan, 2013, p. 114-125.

20. HERES Gerald, Dresdener Kunstsammlungen im 18. Jahrhundert, Leipzig, Seemann, 1991, p. $153-156$.

21. BECK Herbert, BOL P.C., PRINZ W. \& STEUBEN H. von (dir.), Antikensammlungen im 18. Jahrhundert, Berlin, Gebr. Mann, coll. « Frankfurter Forschungen zur Kunst », 1981, p. 273-291.

22. EHRHARDT Wolfgang, Das Akademische Kunstmuseum der Universität Bonn unter der Direktion von Friedrich Gottlieb Welcker und Otto Jahn, Opladen, Westdeutschen Verlag, coll. « Abhandlungen der Rheinisch-Westfälischen Akademie der Wissenschaften », 1982.

23. http://www.abgussmuseum.de/de/abguss-sammlungen-im-ueberblick 
24. FARIAT Axelle, «La situation des académies d'art en Allemagne après 1945 », Regards croisés. Revue franco-allemande de recensions d'histoire de l'art et d'esthétique, $\mathrm{n}^{\circ} 4$, «L'Académie », 2015, p. 69-94 [disponible en ligne], http://hicsa.univ-paris1.fr/documents/pdf/Regards\%20croises/ Regards\%20croises_4/02_7_RC_4_Fariat_fr.pdf [lien valide en novembre 2020].

25. BRINK Sonja \& WISMER Beat, Akademie, Sammlung, Krahe. Eine Künstlersammlung für Künstler, Düsseldorf / Berlin, Museum Kunstpalast / Deutscher Kunstverlag, 2013.

26. Dont une collection de 1200 dessins d'un de ses célèbres directeurs, le sculpteur Johann Gottfried Schadow (1764-1850).

27. https://www.adk.de/de/archiv/

28. Dans les salles de lecture, les bases de données et les catalogues en ligne, les lecteurs peuvent accéder à 13000 mètres linéaires de documents d'archives uniques, environ 1500000 photos, 200000 dessins d'architecture, 75000 œuvres d'art, majoritairement des dessins et près de 50000 affiches.

29. http://www.abguss-sammlung-berlin.de/

30. https://www.smb.museum/museen-und-einrichtungen/gipsformerei/home.html

31. ALTNER Manfred, Dresden. Von der Königlichen Kunstakademie zur Hochschule für Bildende Künste (1764-1989). Die Geschichte einer Institution, Dresde, Verlag der Kunst, 1990.

32. SIMON Robin \& STEVENS Mary Anne, The Royal Academy of Arts. History and collections, New Haven / Londres, Yale University Press, 2018.

33. Voir à ce sujet HOOCK Holger, The King's Artists. The Royal Academy of Arts and the politics of British culture, 1760-1840, Oxford, Clarendon Press, 2003.

34. BARIDON Michel \& OGÉE Frédéric, «Art et nation en Grande-Bretagne : contexte et histoire d'un lien privilégié ", Revue française de civilisation britannique [en ligne], $\mathrm{n}^{\circ}$ 13, vol. 4, 2006, https://journals.openedition.org/rfcb/1627 [lien valide en novembre 2020].w

35. https://www.royalacademy.org.uk/art-artists/search/search-the-collection

36. Qui accueillent en moyenne 250000 visiteurs au $\mathrm{XIX}^{\mathrm{e}}$ siècle.

\section{RÉSUMÉS}

Les écoles d'art des grandes capitales européennes fondées à travers l'Europe, $d u x \mathrm{III}^{\mathrm{e}} \mathrm{au}$ XVIII ${ }^{\mathrm{e}}$ siècle, formulent aujourd'hui les mêmes questions : comment mieux connaitre et mieux valoriser le patrimoine des écoles d'art, et à quelles fins? De l'ouverture d'un nouvel espace muséal à la Royal Academy de Londres en 2016 à celle de la collection anatomique de Dresde en 2018, force est de constater que partout en Europe l'intérêt se porte sur ces collections atypiques dans l'univers muséal, où les chefs-d'œuvre se mêlent aux dessins et plâtres pédagogiques.

Montrer ces collections, c'est donner à voir l'histoire de l'enseignement des artistes, la manière dont «le devenir artiste» s'enseigne, parfois avec des injonctions contradictoires et des approches variées. Les réponses apportées dans les grandes académies européennes diffèrent en raison de leurs histoires, confondues puis séparées de l'institution muséale, et parfois de la section archéologique de l'université. En parcourant l'Europe, de Londres à aux grandes villes allemandes, de Madrid à Vienne en poussant jusqu'à Saint-Pétersbourg, cet article veut offrir un panorama des choix muséographiques opérés afin de valoriser le patrimoine des écoles d'art. Les collections, parfois rapidement qualifiées de « pédagogiques ", témoignent de l'histoire du regard porté sur les modèles italiens puis sur l'art gréco-romain, ou la ténacité du modèle naturaliste. 
C'est ainsi une histoire du regard artiste qui se révèle, comme le montrent les choix opérés à la Royal Academy de Londres.

Today, the art schools of the big European capitals, which were created throughout all Europe from the 17th to the 18th century, are all asking the same questions: how can we better know, and better highlight, the heritage of art schools, and to what end? From the opening of a new museum space in the Royal Academy in London in 2016 to the opening of the Dresden school's anatomy collection in 2018, we are compelled to admit that there is interest all across Europe in these atypical (for the museum world) collections, where known masterpieces are set next to the pedadogical drawings and plaster casts.

To display these collections is to display the history of artist teaching, the way that "becoming an artist" is taught, sometimes with contradictory injunctions and varied approaches. The responses brought by the great European academies are different from each other due to their histories, they were a part of, then separated from the museum institution, and sometimes from the archaeological section of the university. By travelling across Europe, from London to the big German cities, from Madrid to Vienna and even as far as Saint Petersburg, this article aims to show a panorama of the museographic choices that were made to highlight the patrimony of art schools. The collections, which were briefly referred to as "pedagogical", are a testimony of the history of how Italian models, and then greco-roman art, or the tenacity of the naturalist model, were seen. Thus it reveals the history of the artist's way of seeing as shown by the choices made by the Royal Academy of London.

\section{INDEX}

Keywords : art teaching, art school, art academy, art pedagogy, museography, collections, museum, plaster cast gallery, inventory, Vienna Academy, Madrid Academy, Saint Petersburg Academy, London Academy, Royal Academy of Art, San Fernando Academy, Berlin Academy, Dresden Academy

Mots-clés : enseignement artistique, écoles d'art, académie d'art, pédagogie artistique, muséographie, collections, musée, gypsothèque, inventaire, Académie de Vienne, Académie de Madrid, Académie de Saint-Pétersbourg, Académie de Londres, Royal Academy of Art, Académie des beaux-arts de San Fernando, Académie de Berlin, Académie de Dresde

\section{AUTEUR}

\section{MORWENA JOLY-PARVEX}

Conservatrice du patrimoine, Centre des Monuments nationaux

morwena.joly-parvex@monuments-nationaux.fr 